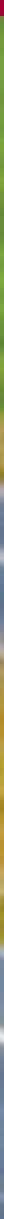

Unfallforschung kompakt

\title{
Sicherheitsanalyse von Straßennetzen: Erfahrungen mit einem automatisierten Verfahren
}

Unfallforschung
der Versicherer

$\neg$ GDV 


\section{Impressum}

Gesamtverband der Deutschen Versicherungswirtschaft e. V.

Unfallforschung der Versicherer

Wilhelmstraße 43/43G, 10117 Berlin

Postfach 0802 64, 10002 Berlin

unfallforschung@gdv.de

www.udv.de

Redaktion: Dipl.-Ing. Jörg Ortlepp

Gestaltung: Michaela Gaebel

Technik: Monika Kratzer-Butenhof

Bildnachweis: Unfallforschung der Versicherer

Erschienen: 10/2011 


\section{Vorbemerkung}

Die Sicherheitsanalyse von Straßennetzen nach dem Regelwerk „Empfehlungen für die Sicherheitsanalyse von Straßennetzen (ESN)“ der Forschungsgesellschaft für Straßen- und Verkehrswesen (FGSV) dient der flächenweiten Identifizierung von Defiziten im Straßennetz und kommt sowohl im Betrieb als auch in der Planung von Straßen zur Anwendung. Aufgrund der flächendeckenden Anwendung müssen erhebliche Datenmengen verarbeitet werden. Daraus ergibt sich die Notwendigkeit dieses Verfahren zu automatisieren.

Das Ziel dieses Projektes war die Umsetzung des ESN-Verfahrens - mit Anwendung der Abschnittsbildung auf Basis der Netzstruktur - in einem Softwaretool sowie die praktische Anwendung in mehreren Bundesländern. Es wurde anhand der Bundesländer Brandenburg, Hessen, NordrheinWestfalen, Sachsen, Sachsen-Anhalt und Thüringen gezeigt, dass dies grundsätzlich möglich ist.

Probleme bestehen noch in der Qualität der Datengrundlage sowie bei der Methodik der Abschnittsbildung. Diese können jedoch teilweise durch Analysemöglichkeiten und Filtereinstellungen in einem Softwaretool behoben werden. 


\section{Inhalt}

Vorbemerkung 2

$1 \quad$ Einleitung 4

2 Eingangsdaten 4

2.1 ESN $2003 \quad 4$

2.2 Unfalldaten 4

2.3 Straßendaten der Länder 4

2.4 Straßennetz 5

2.5 Verkehrsmodell 5

3 Vorgehen zum automatisierten Verfahren 5

3.1 Harmonisierung der Datenquellen 5

3.2 Berechnung der Sicherheitspotenziale nach ESN 8

3.2.1 Berechnung auf Basis der Netzknotenabschnitte 8

3.2.2 Berechnung auf Basis der Navteq-Strecken 8

3.3 Visualisierung der Ergebnisse 9

$4 \quad$ Bewertung der Ergebnisse 11

4.1 Generelle Randbedingungen 11

$\begin{array}{lll}4.2 & \text { Ergebnisse } & 12\end{array}$

$5 \quad$ Fazit und Ausblick 16

$\begin{array}{ll}\text { Literatur } & 18\end{array}$ 


\section{$1 \quad$ Einleitung}

Die Empfehlungen für die Sicherheitsanalyse von Straßennetzen - kurz ESN - enthalten Richtlinien und Berechnungsvorschriften, um das Sicherheitspotenzial von Straßen zu bewerten. Anhand von berichteten Unfalldaten (Kategorie, Lage, Typ, Zeitpunkt, etc.) und empirisch entwickelten, generellen Unfallindikatoren (Unfallkostendichten und -raten sowie Grundunfallkostenraten) wird ein quantitatives Sicherheitspotenzial bestimmt.

Ziel dieses Projektes, das die PTV AG in Karlsruhe für die Unfallforschung der Versicherer (UDV) durchführte, war es, für die ESN ein automatisiertes Verfahren zu entwickeln und für sechs Bundesländer anzuwenden.

\section{Eingangsdaten}

Als Eingangsdaten für die Berechnung der ESN wurden verschiedene Datenquellen herangezogen:

- ESN Dokument Stand 2003

- Unfalldaten aus dem System EUSka (modernes Softwaresystem für die Analyse von Verkehrsunfällen, visualisiert die Unfälle nach den Kriterien der 1-Jahres- und 3-Jahreskarten, filtert das Unfallgeschehen geographisch, nach den Attributen der Unfallanzeige bis hin zu Verkehrsbeteiligung, Alter, Geschlecht der Beteiligten) der Länder Hessen, Nordrhein-Westfalen, Sachsen, Sachsen-Anhalt, Thüringen sowie Unfalldaten aus dem System BASta für Brandenburg

- digitale Straßendaten der o. g. Länder

- bundesweites Straßennetz Navteq

- Verkehrsmodell Validate Deutschland der PTV AG.

\subsection{ESN 2003}

Die ESN als Regelwerk (Empfehlungen für die Sicherheitsanalyse von Straßennetzen,
FGSV 2003) lieferte die Berechnungsvorschriften zur Berechnung der Sicherheitspotenziale auf der Grundlage von Grundunfallkostenraten.

\subsection{Unfalldaten}

Die EUSka Unfalldaten wurden in Form einer MS-Access Datenbank zur Verfügung gestellt. Sie enthält in der Regel die Unfälle der Kategorien 1-6 und der Jahre 2006-2008. Die Unfalldaten enthalten unter anderem Angaben zu:

- Datum

- Straßentyp

- Unfalltyp

- Unfallkategorie

- Koordinaten (WGS84-Bezug) des Unfallorts.

Die Unfalldaten aus Brandenburg wurden als Excel-Datei mit ähnlichen Angaben bereitgestellt. Maßgeblicher Unterschied sind die Lokalisierungsangaben. Für die Unfälle in Brandenburg wurden keine Koordinaten sondern die zugehörigen Netzknotenabschnitte und die Stationierung zur Verfügung gestellt.

\subsection{Straßendaten der Länder}

Die Straßendaten der Länder lagen in Form von mehreren GIS-Layern im MapInfo-Format vor. Für die Straßenklassen (definiert durch die Baulastträgerschaft) Autobahn, Bundesstraße, Landesstraße (Staatsstraße in Sachsen), Kreisstraße und Gemeindestraße stand ein Layer zur Verfügung, welcher folgende relevanten Informationen enthielt:

- Geometrie der Straße (Polylinie)

- Abschnittsbezeichnung

- Straßenklasse (A,B,L/S,K oder G)

- Straßennummer

- Abschnittslänge.

Es gilt dabei zu beachten, dass selbst innerhalb eines Bundeslandes unterschiedliche Informationen zum Straßennetz (z.B. Straßennetzrela- 
tion und Stationierungsrelation) unterschiedliche Aktualität besitzen. Ebenso können sich Unterschiede bezüglich der Aktualität der Kartengrundlage bei der Unfallaufnahme durch die Polizei (z. B. mit EUSka) und den Kartengrundlagen der Straßendaten bei den Baulastträgern ergeben.

\subsection{Straßennetz}

Für das Projekt wurden Navteq Daten für das Bundesgebiet verwendet. Aus allen zur Verfügung stehenden Strecken wurde ein Verkehrsnetz erzeugt, das als Grundlage für alle Berechnungen sowie zur Visualisierung der berechneten Ergebnisse dient, und zwar sowohl auf Basis der Abschnitte der Straßendatenbank der jeweiligen Länder als auch auf Basis der Navteq Strecken. Dafür wurden die Informationen aus den Straßen- und Unfalldatenbanken der Länder auf das Navteq-Netz übertragen.

\subsection{Verkehrsmodell}

Wichtiger Bestandteil der ESN-Berechnungen sind Kenntnisse über den DTV (durchschnittliche tägliche Verkehrsstärke) der Strecken, an denen Unfälle stattgefunden haben. Aus diesem Grund wurden bei dem Projekt bei der Berechnung der ESN das Verkehrsmodell Validate Deutschland der PTV AG mit einbezogen. Dieses liefert flächendeckende DTV-Belastungswerte für das übergeordnete deutsche Straßennetz (Hauptverkehrsstraßen).

Da es auf dem gleichen digitalen Straßennetz von Navteq basiert wie das Verkehrsnetz zur Berechnung der ESN, war es relativ einfach möglich, die DTV-Werte des Modells zur Berechnung der ESN landesweit heran zu ziehen. Grundsätzlich wäre aber auch die Verwendung anderer Quellen für die DTV-Werte (z. B. aus den bundesweiten Straßenverkehrszählungen der Bundesanstalt für Straßenwesen) möglich.

\section{Vorgehen zum automatisierten Verfahren}

\subsection{Harmonisierung der Datenquellen}

Für die Berechnung nach ESN war es notwendig, die Unfälle den Abschnitten der Straßendatenbank der jeweiligen Bundesländer bzw. den entsprechenden Strecken zuzuordnen. Dies wurde mit Hilfe der zur Verfügung stehenden Koordinaten aus der Unfalldatenbank realisiert. Anhand der Angaben zu Koordinaten, Straßenklasse und Straßennummer wurde jeder einzelne Unfall genau einer Navteq Strecke zugeordnet.

Bei der Bestimmung der entsprechenden Strecke wurde berücksichtigt, dass die Koordinaten des Unfalls nicht weiter als $50 \mathrm{~m}$ von der Strecke entfernt lagen und dass sowohl Straßenklasse als auch Straßennummer übereinstimmten. Hierbei wurden die Unfälle auf die aus der Straßendatenbank zugeordneten Daten für Straßenklasse und Straßennummer referenziert. Dieses Vorgehen stellt sicher, dass der Datenstand von Unfalldaten und Netzdaten (aus den Bundesländern) weitgehend übereinstimmt.

Als Ergebnis dieses Zuordnungsschrittes lagen für alle Strecken folgende Informationen vor:

- Anzahl Unfälle im Betrachtungszeitraum (hier drei Jahre)

- Differenzierung der Unfälle nach Unfallkategorien, was die Berechnung von Unfallkosten je Abschnitt ermöglicht.

In Tabelle 1 sind die Zuordnungsraten der Unfälle im Außerortsnetz der sechs untersuchten Bundesländer dargestellt. Aus diesen Zahlen lässt sich folgendes ableiten:

- Anzahl und Anteil der Unfälle, die durch die ESN-Analyse für das klassifizierte Straßennetz außerorts potenziell behandelt werden können (= potenzieller Nutzen einer ESN-Analyse) 
Tabelle 1:

Anzahl und Anteil zugeordneter Unfalldaten (Unfälle aus 2006 bis 2008)

\begin{tabular}{|c|c|c|c|c|c|c|c|}
\hline \multirow[b]{2}{*}{ Sachsen } & \multirow{2}{*}{$\begin{array}{c}\mathbf{U}(\mathbf{P S}) \\
267.442\end{array}$} & \multicolumn{2}{|c|}{ außerorts } & \multicolumn{2}{|c|}{$\begin{array}{c}\text { Straßenklassen } \\
\text { A, B, S (L) }\end{array}$} & \multicolumn{2}{|c|}{$\begin{array}{c}\text { Zuordnung Strecke } \\
\text { möglich / ESN-Analyse }\end{array}$} \\
\hline & & 90.616 & $33,90 \%$ & 69.718 & $76,90 \%$ & 65.082 & $93,40 \%$ \\
\hline Sachsen-Anhalt & 240.880 & 76.623 & $31,80 \%$ & 59.150 & $77,20 \%$ & 53.027 & $89,60 \%$ \\
\hline Hessen & 399.892 & 141.392 & $35,40 \%$ & 124.077 & $87,80 \%$ & 111.073 & $89,50 \%$ \\
\hline Thüringen & 154.085 & 51.660 & $33,50 \%$ & 45.141 & $87,40 \%$ & 36.309 & $80,40 \%$ \\
\hline NR W $^{1)}$ & 510.847 & 88.099 & $17,20 \%$ & 66.348 & $75,30 \%$ & 47.188 & $71,10 \%$ \\
\hline Brandenburg $^{2)}$ & $63.998^{3)}$ & $23.334^{3)}$ & $36,50 \%$ & 11.714 & $50,20 \%$ & 10.507 & $89,7 \%^{4)}$ \\
\hline
\end{tabular}

Anteile in beziehen sich auf den Wert in der jeweils linken Spalte

1) Unfallzeitraum abweichend zu den anderen Bundesländern mit 2007-2009

2) für Brandenburg liegen nur die Unfälle der Kategorien 1 bis 4 und 6 vor (UP,SS),

3) diese Zahlen wurden aus amtlichen Unfallstatistik entnommen (www.DESTATIS.de)

4) dieser Anteil bezieht sich nur auf die abschnittsweise Zuordnung; die Ableitung einer Koordinate war nur für einen Anteil von $84,4 \%$

- Anzahl und Anteil der Unfälle die tatsächlich in die ESN-Analyse eingehen (= tatsächlicher Nutzen einer ESN-Analyse).

Brandenburg stellt in mehrerer Hinsicht einen Sonderfall dar. Die Unfälle stammen aus dem System BASta (siehe auch Höppner \& Wenk 2000 [1]). Dies ist ein auf Maplnfo-Basis aufgebautes Unfall-Analyse-System. Die Verortung der Unfälle erfolgt in Abhängigkeit der Stationierung aus der amtlichen Unfallstatistik und nicht wie bei EUSka direkt bei der Polizei. Aus diesem Grund standen für Brandenburg nur die Unfälle der Kategorien 1 bis 4 und 6 zur Verfügung. Seit 2010 setzt auch die Polizei in Brandenburg EUSka ein.

In Sachsen konnten über 93 \% der Unfälle auf die zu untersuchenden Strecken zugeordnet werden. In Sachsen-Anhalt, Hessen und Brandenburg waren es mit rund $90 \%$ etwas weniger. In Thüringen waren rund $20 \%$ der Unfälle nicht zuzuordnen und in Nordrhein-Westfalen sogar rund $30 \%$, was hier jedoch überwiegend darauf zurückzuführen ist, dass für das Projekt unvollständige Daten verwendet werden mussten.

Die fehlenden Zuordnungen von Unfällen mit Koordinaten zu Streckenabschnitten lassen sich auf folgende Ursachen zurückführen:
- Unfälle ereignen sich auf Rast- und Nebenanlagen von Autobahnen und fallen damit nicht in den 50 m-Korridor (Abbildung 1). Dies ist jedoch unkritisch, da die Unfälle auch inhaltlich nicht mit der eigentlich bewerteten Straße im Zusammenhang stehen.

- Unfälle ereignen sich auf den Rampen planfreier Knotenpunkte (Abbildung 1). Die sehr feine Netzgliederung innerhalb von planfreien Knotenpunkten, aber auch die Zuordnung über den 50 m-Korridor erschweren die genaue Zuordnung. Sehr kurze Abschnittslänge und fehlende DTV-Angaben für Rampen schränken allerdings die Aussagefähigkeit von eventuell berechneten Sicherheitspotenzialen auf solchen Rampen ein. Der Umgang mit solchen Unfällen muss auch von Seiten des Regelwerks ESN noch genauer beschrieben werden.

- Unterschiedlich aktuelle Kartengrundlagen für Unfallaufnahme und Unfallauswertung führen aufgrund von Umwidmung aber auch Neubau von Strecken zur fehlerhaften Zuordnung von Unfällen (Abbildung 2).

- Erfolgt eine Umwidmung ohne Neubau bzw. Verlegung der Straße während des Betrachtungszeitraums der ESN-Analyse so wird nur ein Teil der Unfälle zugeordnet (Abbildung 3). Dies kann zu einer Unterschätzung der berechneten Unfallkosten und damit des Sicherheitspotenzials führen. 


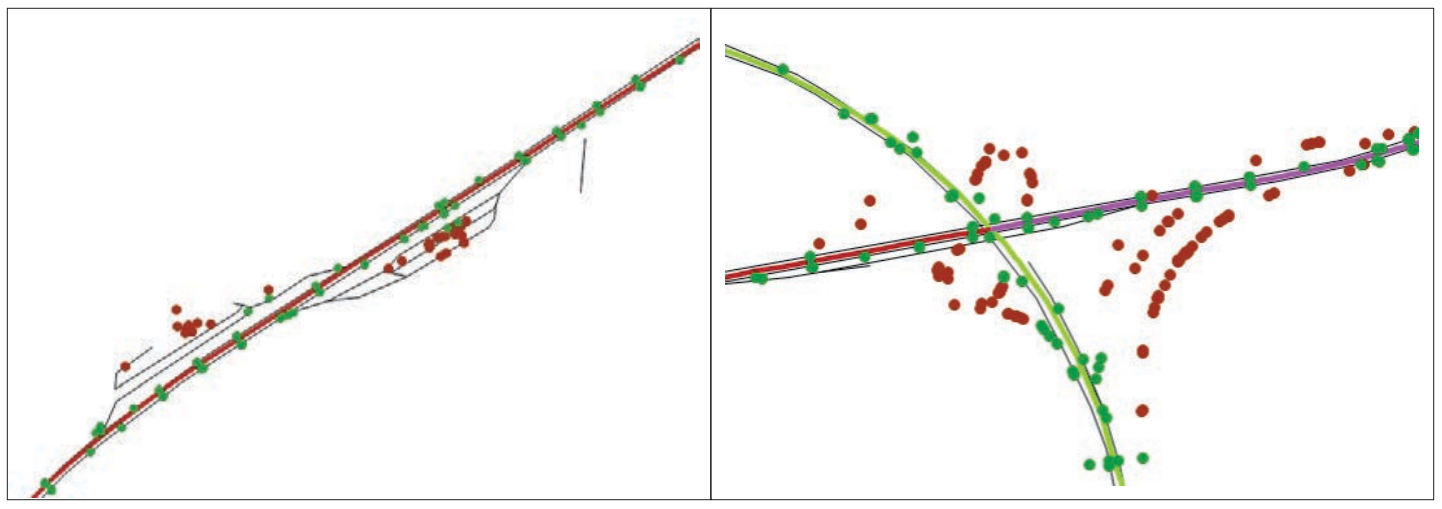

Abbildung 1:

Beispiele für nicht zugeordnete Unfälle auf Nebenanlagen (linkes Bild) und Rampen (rechtes Bild)

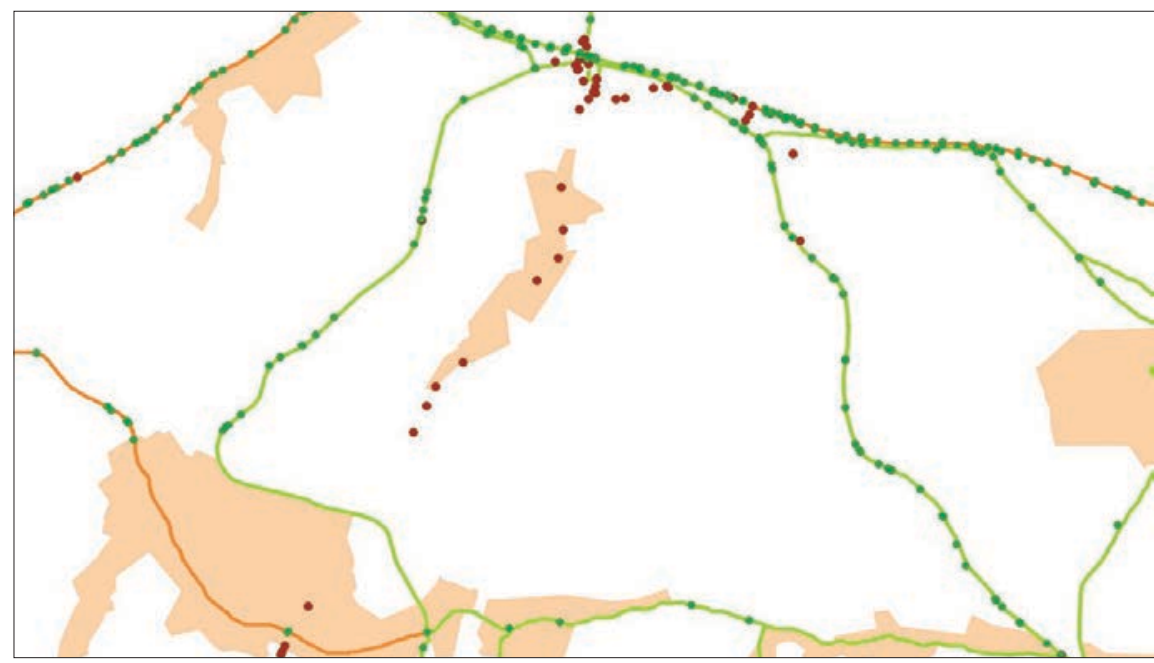

Abbildung 2:

Beispiel für nicht zugeordnete Unfälle (rot gekennzeichnet) durch Bau einer Ortsumgehung und Umwidmung der Ortsdurchfahrt

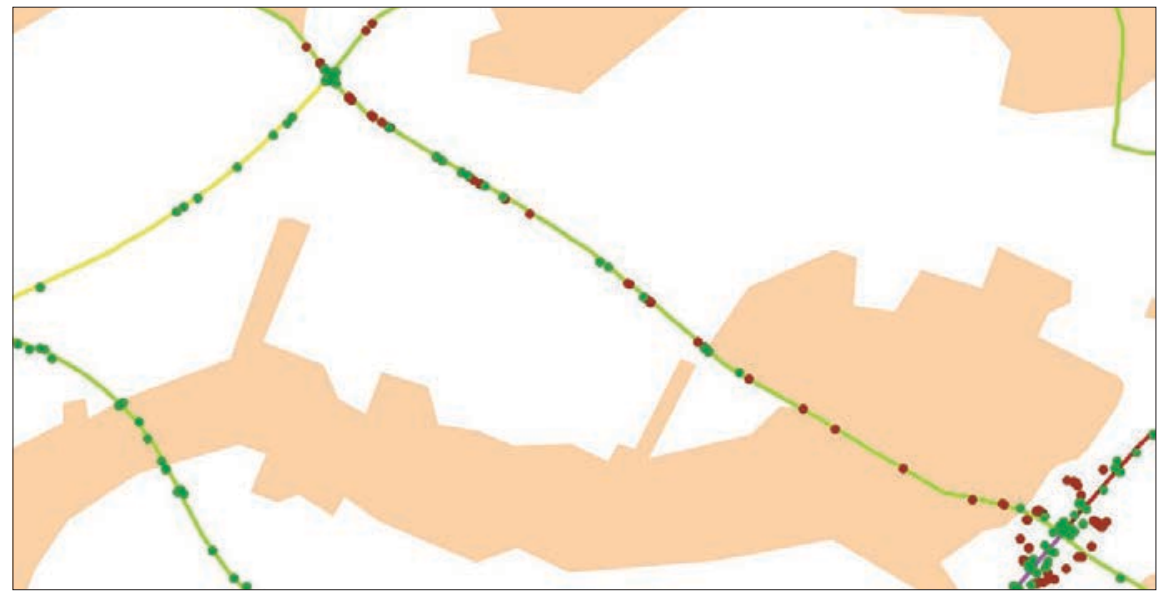

Abbildung 3:

Beispiele für nicht zugeordnete Unfälle (rot gekennzeichnet) durch Umwidmung einer Straße während des Untersuchungszeitraums 


\subsection{Berechnung der Sicherheits- potenziale nach ESN}

Um die Sicherheitspotenziale nach ESN zu berechnen wurden folgende Annahmen getroffen:

- Ein Abschnitt ist ein Element der Straßendatenbank des jeweiligen Landes und entspricht einem Streckenabschnitt zwischen zwei Netzknoten.

- Es wurden nur Unfälle außerorts betrachtet.

- Es wurden nur Unfälle auf Autobahnen, Bundes- und Landesstraßen bzw. StaatsstraBen betrachtet.

- Der Betrachtungszeitraum betrug jeweils drei Jahre.

Die Berechnung der Sicherheitspotenziale findet grundsätzlich auf der Abschnittsebene der digitalen Straßendaten des jeweiligen Landes statt. Um potenzielle Defizite genauer lokalisieren zu können, wurde die Berechnung der Sicherheitspotenziale zusätzlich auf Ebene der Navteq-Abschnitte durchgeführt.

\subsubsection{Berechnung auf Basis der Netzknotenabschnitte}

Zur Berechnung der Sicherheitspotenziale auf Basis der Netzknotenabschnitte wurden zunächst die aus der Zuordnung der Unfälle zu den Navteq Strecken ermittelten Kennwerte (Anzahl Unfälle, Unfallkosten, DTV) anhand der Zugehörigkeit zum Abschnitt aggregiert.

Unfälle an Knoten wurden jeweils der an erster Stelle genannten Straße in der Verkehrsunfallanzeige (VUA) zugeordnet. Dies ist in der Regel die höher klassifizierte Straße, deren Baulastträger zumeist auch für diesen Knotenpunkt zuständig ist. Hier zeigt sich erneut die Notwendigkeit, zusätzlich zur Referenzierung über den 50 m-Korridor auch die Angaben zu Straßenart und Straßennummer heranzuziehen. Eine exaktere Aufteilung der Unfälle z. B. auf die
Zufahrten ist aus Gründen der unzureichenden Datenqualität bzw. fehlenden Informationen in der Unfallanzeige in der Regel nicht möglich.

Die Abschnittslänge für die Berechnung der Sicherheitspotenziale wurde aus der Straßendatenbank des jeweiligen Landes entnommen.

Der DTV-Wert eines Abschnittes wurde als längengewichteter Mittelwert der DTV-Werte der Navteq Strecken ermittelt. Das entspricht dem Vorgehen nach ESN.

Damit lagen für jeden Abschnitt die für die Berechnung der Sicherheitspotenziale (SIPO) relevanten Informationen vor. Die Berechnung selbst erfolgte durch die Anwendung des Formelwerks der ESN. Für beide Fahrtrichtungen einer Strecke wurden die SIPO symmetrisch ermittelt, d.h. die ESN-Ergebnisse beziehen sich auf den Querschnitt und sind für beide Fahrtrichtungen identisch. Eine Auswertung getrennt nach Fahrtrichtungen wäre für baulich getrennte Streckenabschnitte zwar möglich, wurde aber im Rahmen des Projektes nicht durchgeführt.

\subsubsection{Berechnung auf Basis der Navteq- Strecken}

Für die Berechnung der Sicherheitspotenziale auf den einzelnen Navteq-Abschnitten wurden ebenfalls die Formeln nach ESN angewendet. Hierbei wurden auch Abschnitte mit weniger als 300 Meter Länge zur Berechnung herangezogen, um eine lückenlose Berechnung sicher zu stellen. Die SIPO wurden für beide Richtungen einer Navteq-Strecke symmetrisch berechnet, sie beziehen sich somit auf den Querschnitt und sind für beide Fahrtrichtungen identisch. Die Ergebnisse sind als Indikatoren zu verstehen. Sie zeigen, an welchen Stellen innerhalb eines Abschnitts mögliche Defizite lokalisiert sind und geben damit eine erste Hilfestellung für die Weiterverwendung und tiefergehende 
Analyse der Ergebnisse des ESN-Verfahrens bis hin zur örtlichen Unfallanalyse. Die Berechnung der SIPO auf Basis der Navteq-Strecken ist keinesfalls für eine Rangfolgenbildung geeignet. Dazu sind die Abschnitte in der Regel zu kurz und weisen zu geringe Unfallzahlen auf.

\subsection{Visualisierung der Ergebnisse}

Die Visualisierung der Berechnungsergebnisse erfolgt mit handelsüblicher Software. Hierbei können die Ergebnisse auf Basis der Netzknotenabschnitte und der Navteq-Abschnitte einzeln oder gemeinsam dargestellt werden.

Abbildung 4 und Abbildung 5 zeigen typische Beispiele für die Anwendung der Visualisierung. Auf Basis der Netzknotenabschnitte werden zunächst Sicherheitspotenziale berechnet, die eine Priorität für die weitere Vorgehensweise mit diesem Straßenzug vorgeben. Sollen dann erste Ansätze für Maßnahmen zur Verbesserung der Unfallsituation gefunden werden, hilft die Darstellung über die Navteq-Strecken, um die Problemstellen näher einzugrenzen.
Außerdem ist durch die feine Betrachtung der Navteq Strecken auch eine Überprüfung der Aussagefähigkeit bzw. Qualität der Ergebnisse möglich. Ergibt sich beispielsweise ein hohes Sicherheitspotenzial nur aus einer Vielzahl von Unfällen an einer Kurve, ist die Zuordnung des Sicherheitspotenzials zum Streckenzug zumindest in Frage gestellt. Maßnahmen müssten in diesem Fall nur für die Kurve, aber nicht für den gesamten Straßenzug gefunden werden.

Die den Abschnitten zugrunde liegenden Daten lassen sich grundsätzlich auch für weitere Darstellungszwecke nutzen. Über unterschiedliche Grafikparameter und Filtereinstellungen könnten z.B. folgende Inhalte dargestellt werden: - Kenngrößen zur Beschreibung der Unfallzahl oder der Anzahl an nicht zugeordneten Unfällen, um eine verbesserte Beurteilung der Aussagefähigkeit der Bewertung eines Abschnitts zu ermöglichen

- Sicherheitspotenziale direkt für unterschiedliche Straßenklassen

- Einzelunfälle unterschiedlicher Ausprägung, um Informationen für die Ursachen eines

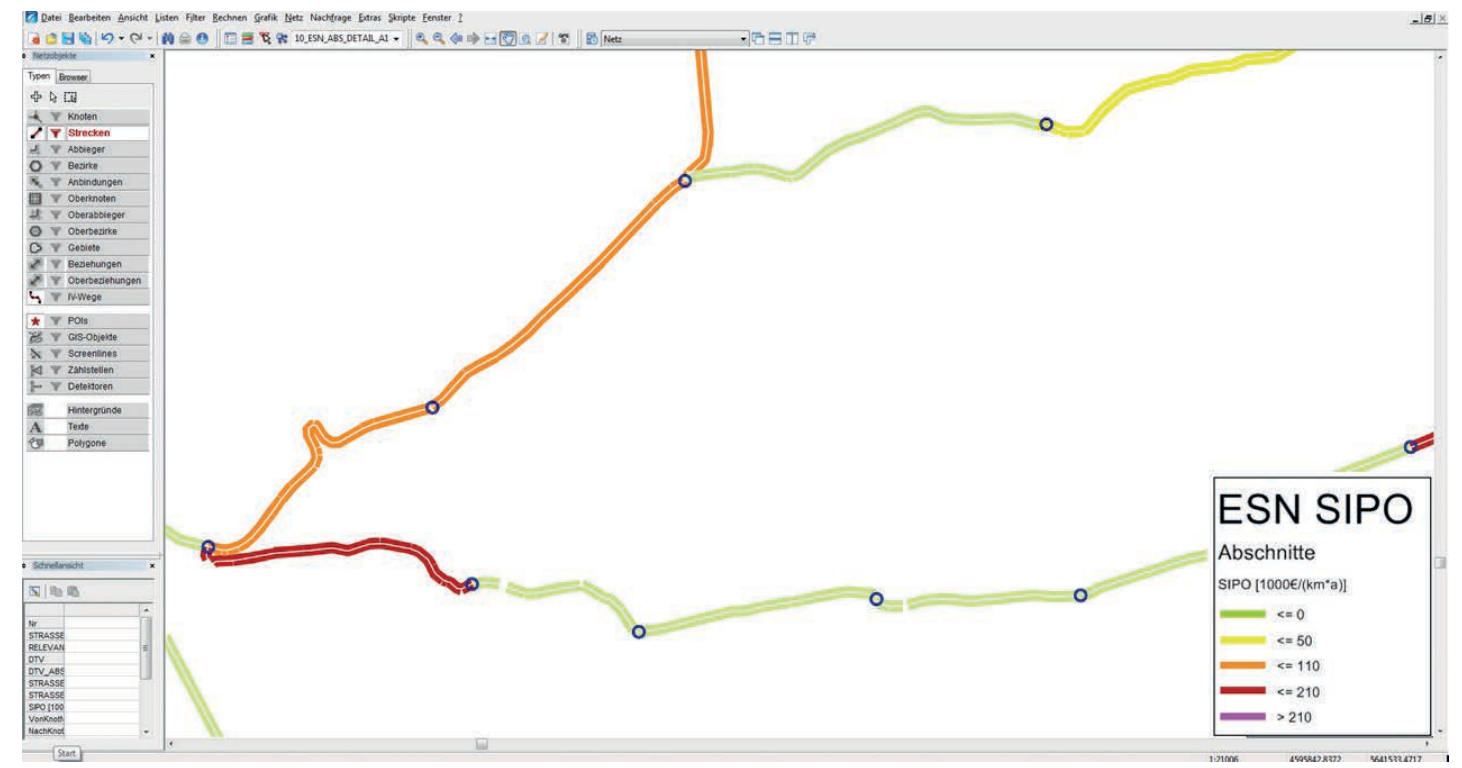

Abbildung 4:

Darstellung der Ergebnisse auf Basis der Netzknotenabschnitte. Blaue Kreise stellen Netzknoten dar. 


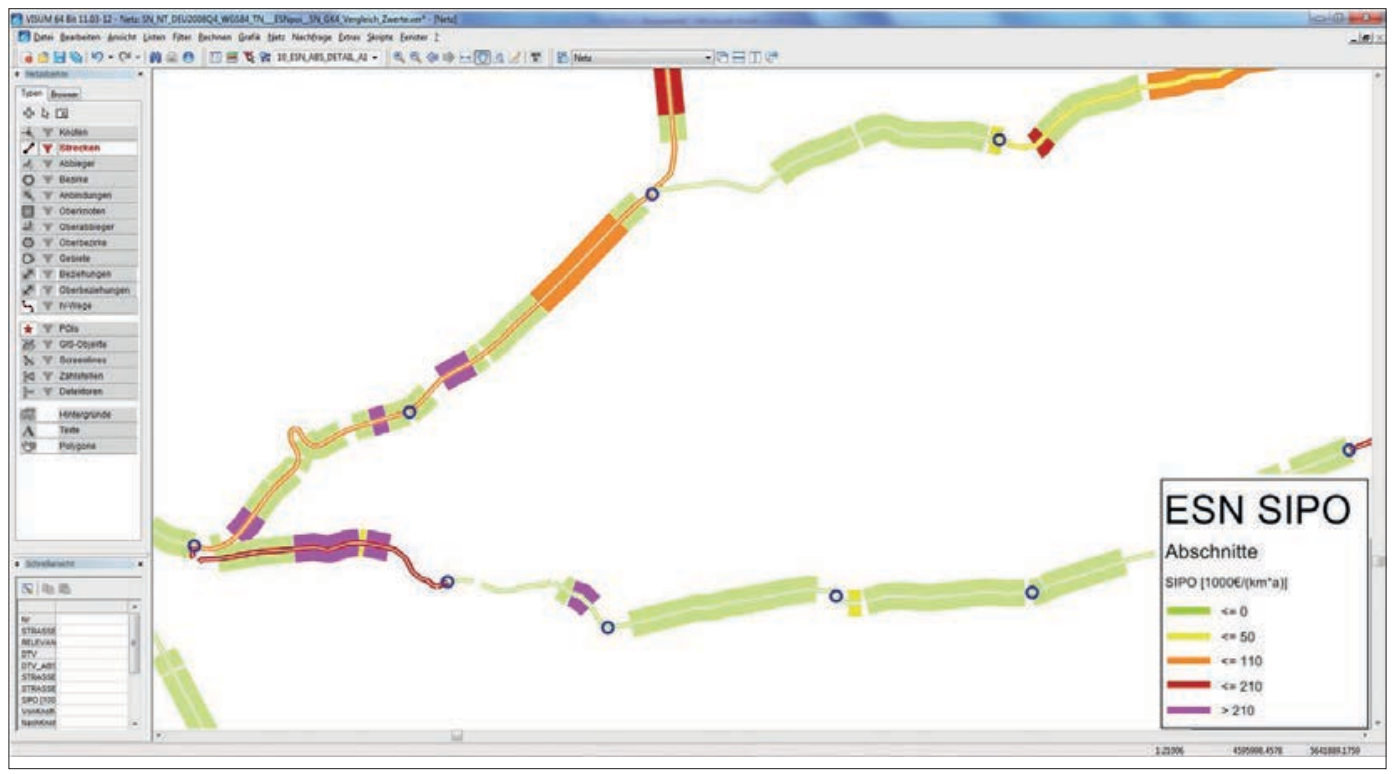

Abbildung 5:

Gemeinsame Ansicht der Ergebnisse auf Netzknotenabschnitten und Navteq-Strecken. Der äußere Balken stellt die Ergebnisse auf Basis der Navteq-Strecken dar. Die Breite des äußeren Balkens modelliert die Anzahl Unfälle. Blaue Kreise stellen Netzknoten dar.

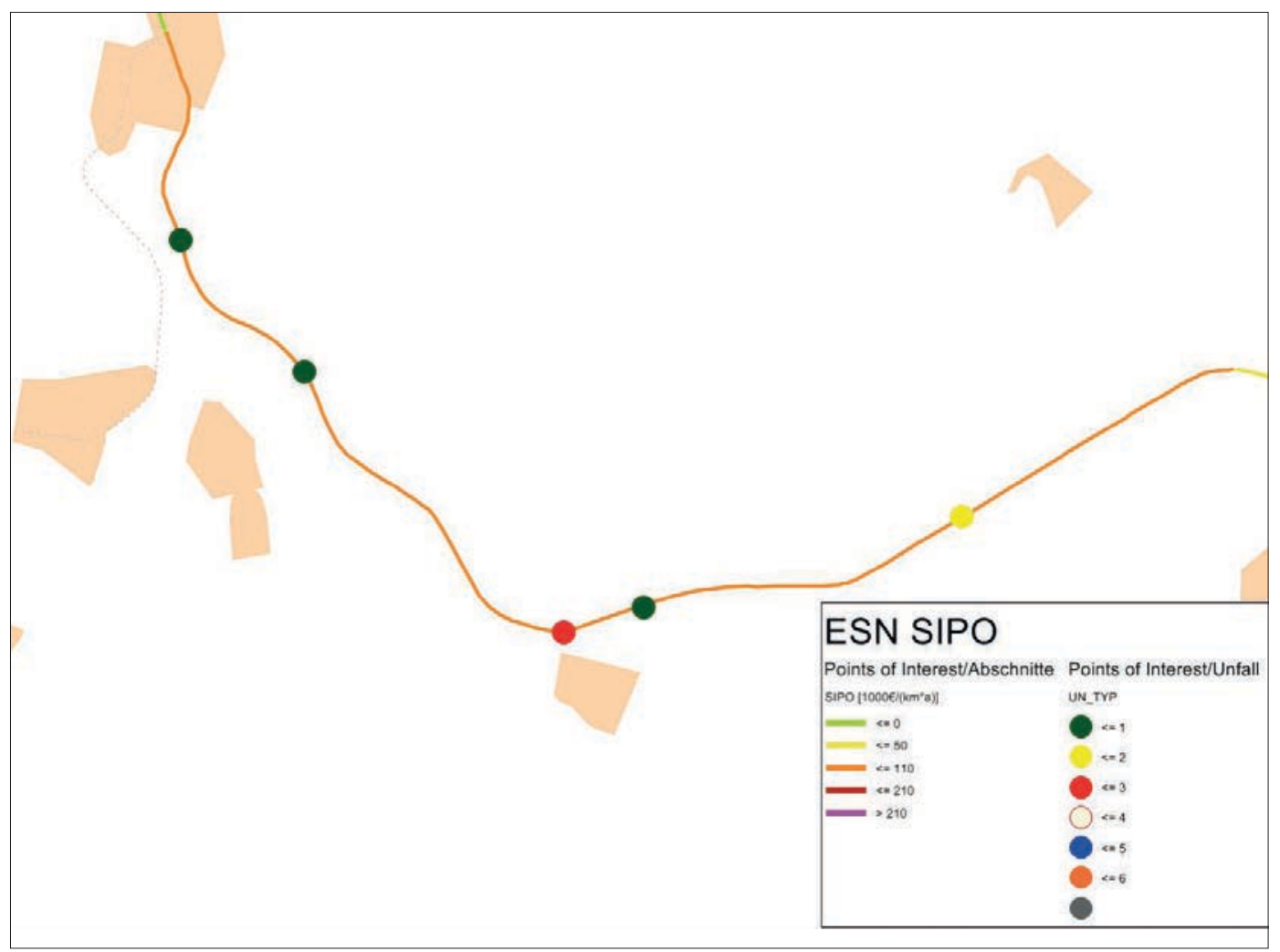

Abbildung 6:

Möglichkeit der gleichzeitigen Visualisierung von Sicherheitspotenzial und Unfälle differenziert nach Unfalltyp (im Bild sind nur Unfälle mit Personenschaden dargestellt) 
hohen Sicherheitspotenzials zu identifizieren (Abbildung 6)

- Abschnitte mit einem hohen Sicherheitspotenzial, die den Anforderungen an lange Abschnittslängen und hohe Unfallzahlen genügen. Dabei würden kurze Abschnitte bzw. Abschnitte mit einer geringen Anzahl an Unfällen mit schwerem Personenschaden ausgeblendet.

\section{Bewertung der Ergebnisse}

\subsection{Generelle Randbedingungen}

Eine flächenhafte automatisierte Bewertung des klassifizierten außerörtlichen Straßennetzes ist in allen betrachteten Bundesländern grundsätzlich möglich. Die praktische Anwendung der ESN 2003 auf Netzknotenbasis ist mit den Einschränkungen aus der zum Teil begrenzten Unfallzuordnung ebenso möglich. Die Zuordnungsprobleme können nur durch aktuelle und aufeinander abgestimmte Datengrundlagen gelöst werden. Den Möglichkeiten, im Rahmen eines automatisierten Verfahrens Zuordnungsprobleme ohne aufwendige manuelle Nacharbeit abzufangen, sind dabei Grenzen gesetzt.

Zudem ist in den ESN bislang nicht hinreichend festgelegt, wie mit Ortsdurchfahrten umzugehen ist. Grundsätzlich sind Innerortsstraßen anders - zum Beispiel mit einer anderen Grundunfallkostenrate - zu bewerten als Außerortsstraßen. Bei der Betrachtung von Netzknotenabschnitten ist vielfach nicht zu vermeiden, dass ein Teilabschnitt von der Ortsgrenze bis zum ersten innerörtlichen Netzknoten oder in kleineren Orten sogar die gesamte Ortsdurchfahrt im Innerortsbereich liegen (Abbildung 7). Das bedeutet, dass die Länge des Netzknotenabschnitts auch innerörtliche Abschnitte enthalten kann, und damit zu ungenauen Ergebnissen für das Sicherheitspotenzial dieser Abschnitte führt. Würde man aber alle
Innerortsbereiche ausklammern, könnte das sehr kurze Abschnittslängen zur Folge haben. Das Problem der kurzen Abschnittslängen und kleinen Unfallzahlen ist umfassend in Ebersbach \& Schüller 2008 [2] dokumentiert. Kurze Abschnitte und/oder Abschnitte mit kleinen Unfallzahlen können zu unzutreffenden Ergebnissen führen und vor allem die Höhe des Sicherheitspotenzials und die Prioritäten in der Rangfolge erheblich beeinflussen. Bisher existieren keine abgestimmten Vorschläge wie solche Abschnitte vermieden bzw. von vornherein (also bei der Abschnittsbildung) ausgeschlossen werden können. Es ist aber möglich zumindest die Auswirkungen dieses Problems zu reduzieren:

- Weisen Abschnitte eine kürzere Länge als eine definierte Mindestlänge (zum Beispiel von $1 \mathrm{~km}$ ) auf, wird ihnen eine virtuelle Länge in Höhe der Mindestlänge zugewiesen. Damit werden die negativen Auswirkungen kurzer Längen nivelliert.

- Weist ein Abschnitt weniger als eine Mindestanzahl an Unfällen auf, wird diesem Abschnitt ein Sicherheitspotenzial von 0 (1000 €/km*a) zugewiesen. Die Mindestanzahl an Unfällen kann unterschiedlich definiert werden. Die ESN geht von $4 \mathrm{U}(\mathrm{S})$ je $A b$ schnitt in 3 Jahren aus, Ebersbach \& Schüller schlagen $1 \mathrm{U}(\mathrm{SP}) /\left(3 \mathrm{a}^{*} \mathrm{~km}\right)$ vor.

Beide Maßnahmen sorgen dafür, dass tatsächlich ungerechtfertigte Sicherheitspotenziale nicht mehr eine hohe Priorität in der Rangfolge erhalten und verbessern damit die Aussagefähigkeit der flächenhaften Analyse. Beide Maßnahmen lassen sich mit dem hier vorgestellten Tool umsetzen.

Da bisher aber noch keine abschließende einheitliche Meinung zu diesem Sachverhalt existiert, erfolgte die Berechnung der Sicherheitspotenziale ausschließlich nach den Vorgaben der ESN. 
Das Sicherheitspotenzial auf Abschnitten mit Innerortsbereichen stellt daher eine Unterschätzung des tatsächlichen Wertes dar, da Unfälle innerorts nicht betrachtet werden, die innerörtliche Streckenlänge aber trotzdem in die Berechnung des Sicherheitspotenzials eingeht. Dieser Sachverhalt muss bei der Bewertung der einzelnen Abschnitte vom jeweiligen Bearbeiter entsprechend berücksichtigt werden. Hier sind der automatisierten Bewertung derzeit noch Grenzen gesetzt. Theoretisch könnten jedoch auch die Ortsgrenzen (Beginn der Ortsdurchfahrt) als „virtuelle Netzknoten“ betrachtet werden, wenn die Abschnitte dadurch nicht zu klein werden. Hierzu fehlt aber bislang ebenfalls ein einheitliches Vorgehen.

\subsection{Ergebnisse}

In Sachsen konnten 93,4 \% der Unfälle den Streckenabschnitten zugeordnet werden (Tabelle 2). Die Ergebnisse können somit als eine Art Referenz für die praktische Anwendung der ESN gesehen werden. Im Folgenden wird daher insbesondere auf die Ergebnisse in Sachsen eingegangen.

Erwartungsgemäß weisen Autobahnabschnitte die größten Längen und die höchsten Unfalldichten je Kilometer und je Abschnitt auf (Tabelle 2). Für nahezu das gesamte Autobahnnetz liegen DTV-Angaben vor. Die Anwendung der Analyse und Interpretation der Ergebnisse

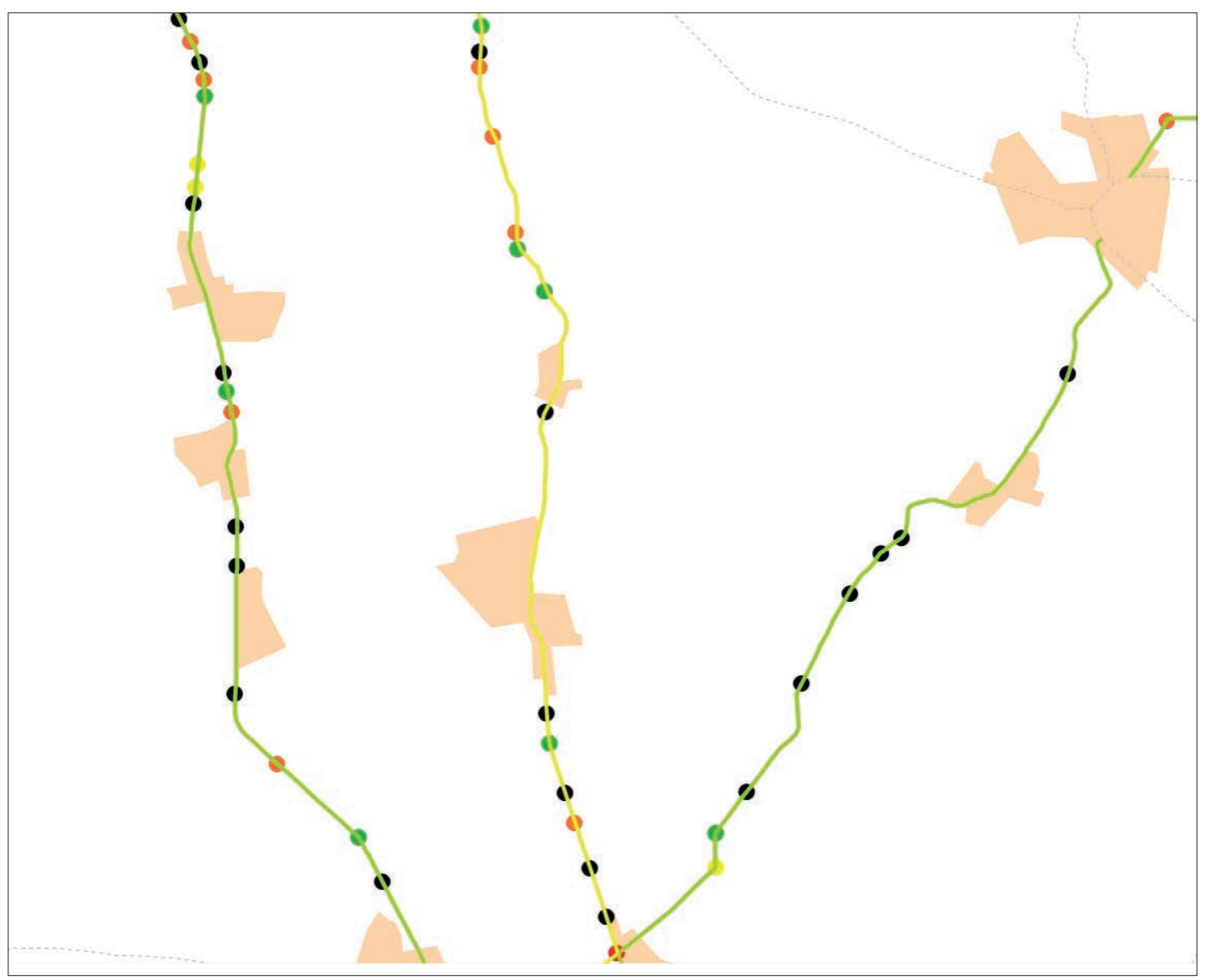

Abbildung 7:

Darstellung zur Problematik der Berücksichtigung von Ortsdurchfahrten (Länge der ODs gehen in die Berechnung des SIPOs ein, Unfälle auf OD werden nicht berücksichtigt) 
ist damit für Autobahnabschnitte uneingeschränkt möglich.

Auf den Bundes- und Staatsstraßen sind sowohl die mittleren Abschnittslängen als auch die Unfalldichten - vor allem bezogen auf die Unfälle mit schwerem Personenschaden U(SP) geringer.

In allen Bundesländern zeigt sich die Tendenz, dass der Anteil an Strecken ohne Datenversorgung mit abnehmender Klassifizierung der Straße ansteigt. Auf Autobahnen lag der Anteil zwischen $1 \%$ und $15 \%$, auf BundesstraBen zwischen $6 \%$ und $15 \%$ und auf Landes-/ Staatsstraßen zwischen $5 \%$ und $33 \%$. Für diese Abschnitte erfolgte im Rahmen des Projektes keine Bewertung bzw. Berechnung eines Sicherheitspotenzials. Es gilt aber zu beachten, dass die Strecken ohne Datenversorgung ein heterogenes Kollektiv darstellen. Das sind einerseits Strecken, die aufgrund einer ungenügenden Zuordnungsrate keine Unfälle oder Angaben zum DTV enthalten. Gründe dafür sind bspw. die Umwidmung, der Neubau oder die Lage der Strecke innerorts (es wurden nur Unfälle auf Außerortsstraßen berücksichtigt). Andererseits wurden aber auch Strecken nicht bewertet, die tatsächlich keine Unfälle aufweisen und somit eigentlich ein negatives Sicherheitspotenzial bzw. ein Sicherheitspotenzial von Null erhalten müssten. Der Grund dafür ist, dass sich beide Typen von Strecken ohne Datenversorgung nur schwer abgrenzen lassen. Der tatsächliche Anteil der Strecken ohne Datenversorgung liegt somit etwas unter dem angegebenen Wert.

Der Anteil sehr kurzer Abschnittslängen (hier $<1 \mathrm{~km}$ ) der gesamten Straßennetzlänge der jeweiligen Straßenkategorie liegt überall unter $13 \%$. Das bedeutet, der überwiegende

Tabelle 2:

Kenngrößen zum Straßennetz und Unfallgeschehen differenziert nach Straßenklassen (Beispiel Sachsen)

\begin{tabular}{|c|c|c|c|c|c|c|}
\hline & & & $\begin{array}{c}\text { Bundes- } \\
\text { autobahnen }\end{array}$ & Bundesstraßen & $\begin{array}{l}\text { Staatsstraßen/ } \\
\text { Landesstraßen }\end{array}$ & Kreisstraßen \\
\hline \multicolumn{2}{|c|}{ Straßennetzlänge } & {$[\mathrm{km}]$} & 541 & 2.421 & 4.762 & 5.884 \\
\hline \multicolumn{2}{|c|}{ Anzahl Abschnitte } & {$[-]$} & 108 & 1.295 & 2.296 & 2.577 \\
\hline \multicolumn{3}{|c|}{$\begin{array}{c}\text { Anteil Straßennetzlänge } \\
\text { ohne DTV-Angaben }\end{array}$} & $<1 \%$ & $10 \%$ & $13 \%$ & - \\
\hline \multicolumn{2}{|c|}{$\varnothing$ Abschnittslänge } & {$[\mathrm{km}]$} & 5,0 & 1,9 & 2,1 & 2,3 \\
\hline \multicolumn{3}{|c|}{$\begin{array}{l}\text { Anteil Abschnitte } \\
\text { mit } L<1 \mathrm{~km} \text { bez. auf } \\
\text { Straßennetzlänge }\end{array}$} & $1 \%$ & $10 \%$ & $8 \%$ & $5 \%$ \\
\hline & $\mathrm{U}(\mathrm{SP})$ & {$[\mathrm{U} / 3 \mathrm{a}]$} & 532 & 1.393 & 1.711 & 0 \\
\hline & $U(P)$ & {$[\mathrm{U} / 3 a]$} & 1.668 & 3.659 & 4.137 & 0 \\
\hline & $\mathrm{U}(\mathrm{S})$ & {$[U / 3 a]$} & 14.468 & 19.506 & 21.690 & 0 \\
\hline \multirow{2}{*}{$\mathrm{U}(\mathrm{SP})$} & je Kilometer & {$[\mathrm{U} / \mathrm{km} * 3 \mathrm{a}]$} & 1,0 & 0,6 & 0,4 & - \\
\hline & je Abschnitt & {$[U / 3 a]$} & 4,9 & 1,1 & 0,7 & - \\
\hline \multirow{2}{*}{$U(P)$} & je Kilometer & {$[\mathrm{U} / \mathrm{km} * 3 \mathrm{a}]$} & 3,1 & 1,5 & 0,9 & - \\
\hline & je Abschnitt & {$[U / 3 a]$} & 15,4 & 2,8 & 1,8 & - \\
\hline
\end{tabular}

U: Unfälle; U(S): Unfälle mit Sachschaden; U(P): Unfälle mit Personenschaden; U(SP): Unfälle mit schwerem Personenschaden 
Teil des Straßennetzes besitzt Abschnitte mit ausreichenden Längen für eine aussagefähige Bewertung des Sicherheitspotenzials.

Die Verteilung des Sicherheitspotenzials bzw. der daraus berechneten absolut vermeidbaren Unfallkosten lässt sich auch über Lorenzkurven darstellen (Abbildung 8). Dabei werden die vermeidbaren Unfallkosten (vUK) der Länge des jeweiligen Straßennetzes gegenübergestellt. Die sehr starke Konzentration der vermeidbaren Unfallkosten in Abbildung 8 (ca. 70 \% vUK auf $10 \%$ der Netzlänge) ist darauf zurückführen, dass hier die Ortsdurchfahrten längenmäßig in die Untersuchung eingehen, aber nicht die dazugehörigen Unfälle. Damit vergrößert sich der Anteil des Straßennetzes ohne Sicherheitspotenzial und die Lorenzkurve verschiebt sich nach links im Diagramm.

Die Verwendung und Interpretation von Lorenzkurven sollte daher mit großer Vorsicht erfolgen. Hohe Konzentrationen von Sicherheitspotenzialen auf einem geringen Anteil des Straßennetzes werden neben dem oben genannten Einfluss auch durch im Durchschnitt kurze Abschnittslängen begünstigt. Je kürzer die Abschnittslängen sind - bei gleichbleibender Verteilung der Unfälle und "tatsächlicher" Sicherheitspotenziale - umso wei-

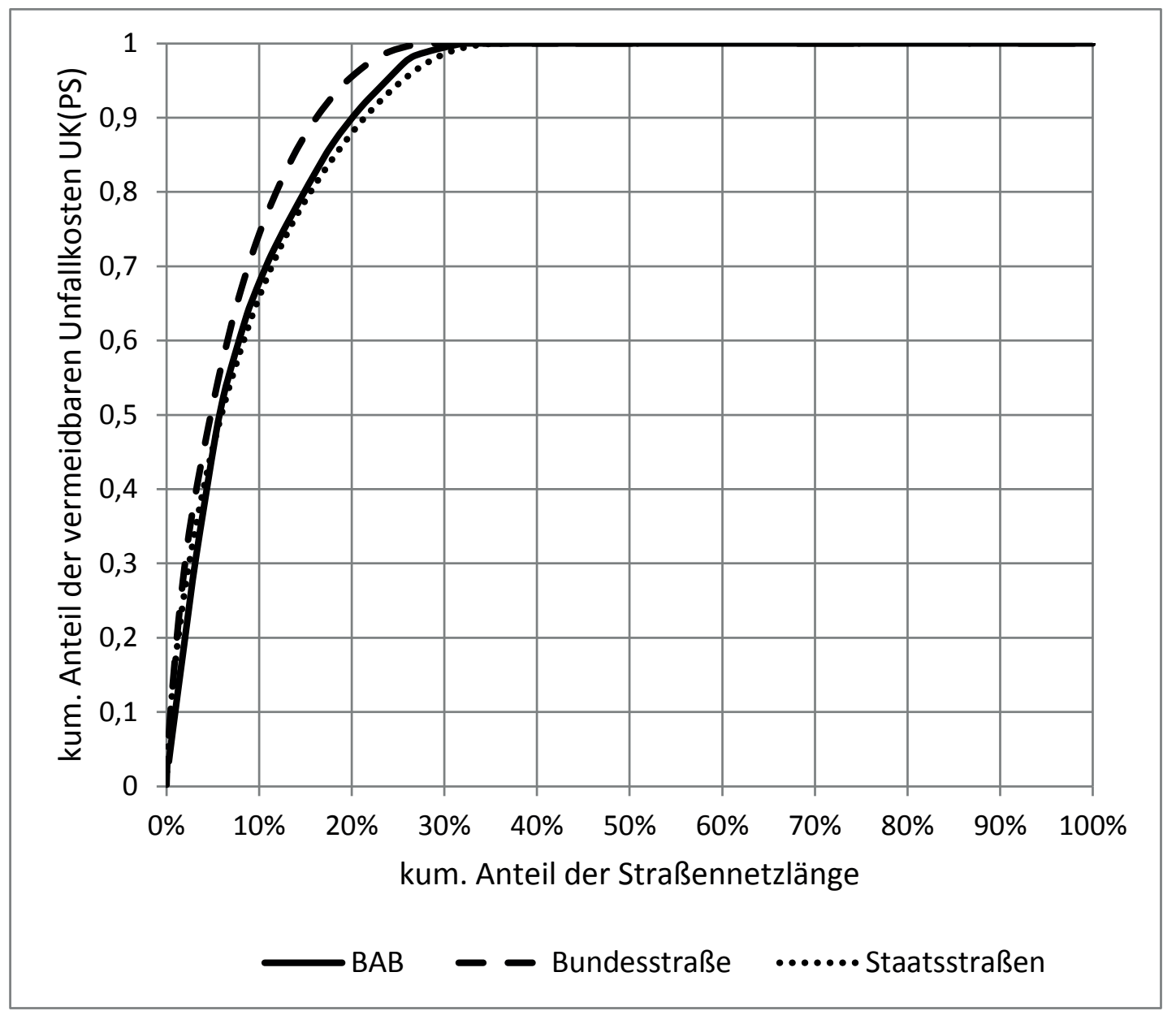

Abbildung 8:

Lorenzkurven der vermeidbaren Unfallkosten in Abhängigkeit der Netzlänge für die untersuchten Strassenkategorien 
ter verschiebt sich die Lorenzkurve nach links. Eine aussagekräftige ESN-Analyse stellt dies dann nicht mehr dar.

In Rangfolgelisten werden die Abschnitte nach Höhe der Sicherheitspotenziale sortiert aufgelistet (Abbildung 9).

Erwartungsgemäß sind die oberen Plätze mit Abschnitten besetzt, die eine kurze Länge und nur eine geringe Zahl an Unfällen mit schwerem Personenschaden aufweisen. Die Aussagefähigkeit dieser Abschnitte bezüglich ihres Sicherheitspotenzials ist eingeschränkt. Um dies zu beheben wurde allen Abschnitten mit einer Länge von < 1 km eine „virtuelle“ Länge von $1 \mathrm{~km}$ für die Berechnung des Sicherheitspotenzials zugewiesen (entsprechend der vorgeschlagenen Methodik in Ebersbach \& Schül- ler 2008 [2]). Aus dem so berechneten modifizierten Sicherheitspotenzial (s. Abbildung 10) wurde eine neue Rangfolge gebildet und der ursprünglichen gegenübergestellt.

Es zeigt sich folgendes Ergebnis:

- Es treten nur noch vereinzelt kurze Abschnitte aber auch Abschnitte mit einer geringen Zahl an U(SP) auf den oberen Plätzen auf (obwohl der Filter sich nur auf die Längen bezieht).

- Der Anteil vermeidbare Unfallkosten konzentriert sich nach Anwendung des Filters auf deutlich weniger Streckenabschnitte. Neben einer Verbesserung der Aussagefähigkeit der hochprioritären Abschnitte ist auch eine verstärkte Konzentration des Sicherheitspotenzials auf weniger, dafür aber langen Abschnitten mit ausreichend hohen Unfallzahlen $\mathrm{U}(\mathrm{SP})$ feststellbar.

\begin{tabular}{|c|c|c|c|c|c|c|c|c|c|c|c|c|}
\hline STRASSENART & DTV & LAENGE & UN_KAT_1 & UN_KAT_2 & UN_KAT_3 & UN_KAT_4 & UN_KAT_5 & UN_KAT_6 & $\mathrm{U}(\mathrm{SP})$ & KOSTEN & SIPO & Rang \\
\hline B & 11488 & 64 & 0 & 1 & 0 & 0 & 9 & 0 & 1 & 334.000 & 1593 & 1 \\
\hline B & 7691 & 30 & 0 & 0 & 2 & 2 & 7 & 0 & 0 & 104.000 & 1057 & 2 \\
\hline B & 12654 & 398 & 0 & 3 & 3 & 8 & 28 & 0 & 3 & 1.166 .000 & 815 & 3 \\
\hline B & 2295 & 644 & 0 & 4 & 6 & 4 & 12 & 0 & 4 & 1.352 .000 & 670 & 4 \\
\hline B & 12245 & 274 & 1 & 1 & 1 & 1 & 9 & 0 & 2 & 645.000 & 628 & 5 \\
\hline B & 8210 & 453 & 0 & 3 & 0 & 0 & 18 & 0 & 3 & 948.000 & 593 & 6 \\
\hline B & 5762 & 130 & 0 & 0 & 4 & 5 & 18 & 0 & 0 & 245.000 & 555 & 7 \\
\hline B & 26796 & 750 & 0 & 6 & 4 & 4 & 21 & 0 & 6 & 1.930 .000 & 515 & 8 \\
\hline B & 14878 & 1105 & 1 & 6 & 0 & 0 & 4 & 0 & 7 & 1.984 .000 & 408 & 9 \\
\hline B & 8360 & 1372 & 0 & 7 & 3 & 3 & 9 & 0 & 7 & 2.107 .000 & 405 & 10 \\
\hline B & 13766 & 642 & 0 & 2 & 12 & 4 & 45 & 0 & 2 & 1.098 .000 & 394 & 11 \\
\hline B & 16156 & 860 & 0 & 4 & 6 & 7 & 17 & 1 & 4 & 1.434 .000 & 349 & 12 \\
\hline B & 13211 & 1283 & 0 & 6 & 7 & 1 & 29 & 0 & 6 & 1.993 .000 & 349 & 13 \\
\hline B & 4010 & 1089 & 1 & 3 & 4 & 1 & 13 & 0 & 4 & 1.283 .000 & 341 & 14 \\
\hline B & 1745 & 319 & 0 & 1 & 0 & 0 & 7 & 0 & 1 & 322.000 & 314 & 15 \\
\hline
\end{tabular}

Abbildung 9:

\section{Rangfolgelisten nach ESN}

\begin{tabular}{|c|c|c|c|c|c|c|c|c|c|c|c|c|c|c|c|}
\hline STRASSENART & DTV & LAENGE & mod_LAENGE & UN_KAT_1 & UN_KAT_2 & UN_KAT_3 & UN_KAT_4 & UN_KAT_5 & UN_KAT_6 & U(SP) & KOSTEN & SIPO & Rang & mod_SIPO & mod_Rang \\
\hline B & 2295 & 644 & 1000 & 0 & 4 & 6 & 4 & 12 & 0 & 4 & 1.352 .000 & $\mathbf{6 7 0}$ & 4 & $\mathbf{4 2 1}$ & 1 \\
B & 14878 & 1105 & 1105 & 1 & 6 & 0 & 0 & 4 & 0 & 7 & 1.984 .000 & $\mathbf{4 0 8}$ & 9 & $\mathbf{4 0 8}$ & 2 \\
B & 8360 & 1372 & 1372 & 0 & 7 & 3 & 3 & 9 & 0 & 7 & 2.107 .000 & $\mathbf{4 0 5}$ & 10 & $\mathbf{4 0 5}$ & 3 \\
B & 13211 & 1283 & 1283 & 0 & 6 & 7 & 1 & 29 & 0 & 6 & 1.993 .000 & $\mathbf{3 4 9}$ & 13 & $\mathbf{3 4 9}$ & 4 \\
B & 4010 & 1089 & 1089 & 1 & 3 & 4 & 1 & 13 & 0 & 4 & 1.283 .000 & $\mathbf{3 4 1}$ & 14 & $\mathbf{3 4 1}$ & 5 \\
B & 8558 & 1356 & 1356 & 0 & 5 & 3 & 2 & 37 & 0 & 5 & 1.702 .000 & $\mathbf{3 0 9}$ & 16 & $\mathbf{3 0 9}$ & 6 \\
B & 26796 & 750 & 1000 & 0 & 6 & 4 & 4 & 21 & 0 & 6 & 1.930 .000 & $\mathbf{5 1 5}$ & 8 & $\mathbf{3 0 1}$ & 7 \\
B & 16838 & 1507 & 1507 & 1 & 5 & 11 & 15 & 37 & 0 & 6 & 2.295 .000 & $\mathbf{2 9 3}$ & 20 & $\mathbf{2 9 3}$ & 8 \\
B & 12863 & 2073 & 2073 & 1 & 7 & 9 & 5 & 50 & 0 & 8 & 2.767 .000 & $\mathbf{2 8 1}$ & 21 & $\mathbf{2 8 1}$ & 9 \\
B & 5966 & 2711 & 2711 & 0 & 8 & 11 & 8 & 56 & 1 & 8 & 2.891 .000 & $\mathbf{2 7 9}$ & 23 & $\mathbf{2 7 9}$ & 10 \\
B & 4276 & 1888 & 1888 & 1 & 5 & 5 & 1 & 18 & 0 & 6 & 1.891 .000 & $\mathbf{2 7 9}$ & 24 & $\mathbf{2 7 9}$ & 11 \\
B & 7905 & 2064 & 2064 & 0 & 7 & 7 & 6 & 29 & 1 & 7 & 2.351 .000 & $\mathbf{2 7 9}$ & 25 & $\mathbf{2 7 9}$ & 12 \\
B & 16156 & 860 & 1000 & 0 & 4 & 6 & 7 & 17 & 1 & 4 & 1.434 .000 & $\mathbf{3 4 9}$ & 12 & $\mathbf{2 7 2}$ & 13 \\
B & 6457 & 1165 & 1165 & 0 & 4 & 1 & 2 & 6 & 0 & 4 & 1.200 .000 & $\mathbf{2 6 1}$ & 27 & $\mathbf{2 6 1}$ & 14 \\
B & 9085 & 1698 & 1698 & 0 & 6 & 5 & 3 & 18 & 0 & 6 & 1.917 .000 & $\mathbf{2 6 0}$ & 28 & $\mathbf{2 6 0}$ & 15 \\
\hline
\end{tabular}

Abbildung 10: Modifizierte Rangfolgelisten 


\section{$5 \quad$ Fazit und Ausblick}

Durch das in dem Forschungsprojekt entwickelte automatisierte Verfahren können selbst landesweite Netzanalysen nach ESN in relativ kurzer Bearbeitungszeit erstellt und künftig aktualisiert werden. Abbildung 11 zeigt schematisch den Ablauf der automatischen ESNAnalyse.

Das jeweilige Rechenergebnis lässt sich ausgehend von den Analysemechanismen eines mo- dernen grafischen Tools schnell und zielgerichtet analysieren.

Dabei können über ein Ranking auffallende Abschnitte identifiziert und genauer betrachtet werden. Einschränkungen aus der Datenqualität lassen sich schnell eingrenzen und in die Darstellungen und Listen integrieren. Selbst ein Vergleich der Sicherheitspotenziale über mehrere Jahre hinweg lässt sich ausgehend von diesen Komponenten ohne hohen zusätzlichen Aufwand bewerkstelligen.

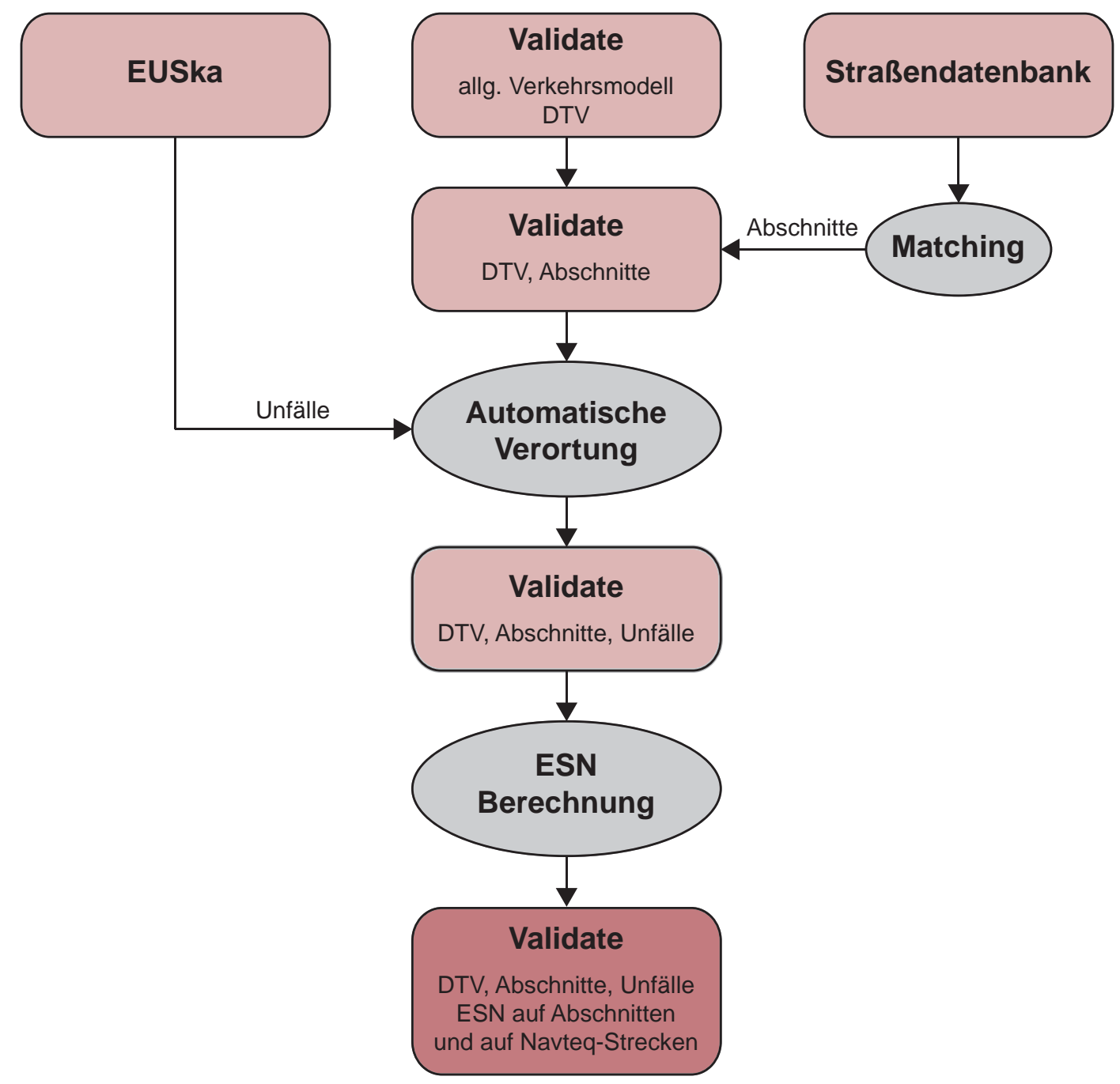

Abbildung 11:

Ablauf der ESN-Analyse mit erforderlichen Eingangsdaten 
Im Rahmen der Umsetzung der ESN-Analyse sind für die Zukunft auch noch weitere Analysemöglichkeiten denkbar:

- Erweiterung der ESN-Analyse auf städtische Straßennetze. Sowohl die linienhafte Bewertung des Hauptverkehrsstraßennetzes als auch der gebietsweise Vergleich von Wohngebieten lassen sich realisieren.

- Die ESN-Analyse kann auch als Verfahren für die Verkehrsplanung eingesetzt werden. Werden Prognosewerte des Verkehrsaufkommens für eine ESN-Analyse mit prognostizierten Sicherheitspotenzialen verwendet, können Änderungen im Netz auch aus Sicht der Verkehrssicherheit bewertet werden.

- Durch die Integration weiterer Unfallmerkmale kann die strukturelle Zusammensetzung des Sicherheitspotenzials bezüglich Unfalltyp, Verkehrsbeteiligung oder weiterer Merkmale näher analysiert werden. Die ESN bietet beispielsweise Vergleichswerte für Unfallkostenbeiträge von Unfalltypen, Unfallarten oder Verkehrsbeteiligungen. Denkbar wäre eine Visualisierung dieser Ko- stenstruktur anhand von Diagrammen, die in einer kleinmaßstäblichen Karte streckenspezifisch dargestellt werden könnten.

- Auch wenn die ESN ein grundsätzlich anderes Verfahren darstellt als die Örtliche Unfalluntersuchung, werden im Ergebnis Bereiche für Maßnahmen zur Verbesserung der Verkehrssicherheit identifiziert. Damit es zu keinen Überschneidungen kommt, sollten zumindest bearbeitete und mit Maßnahmen versehene Bereiche des Straßennetzes über Schnittstellen in beiden Tools (EUSka und grafisches Tool) abgeglichen werden.

Anhand des hier vorgestellten Projektes wurde deutlich, dass eine flächendeckende ESNAnalyse nach dem derzeitigen Stand der ESN (FSGV 2003) auch bei unterschiedlichen Rahmenbedingungen möglich ist. Die Verfügbarkeit von Daten in hinreichender Qualität sowie Vorgaben zum Umgang mit kurzen Abschnitten und innerörtlichen Netzknotenabschnitten müssen jedoch noch einheitlich geregelt werden. 


\section{Literatur}

Höppner, M.; Wenk, S. (2000). BASta - Expertensystem zur digitalen Unfall-Auswertung im Land Brandenburg. In: Straßenverkehrstechnik, (10), S. 534ff

Ebersbach, D.; Schüller, H. (2008). Praktische Anwendung der Empfehlungen für die Sicherheitsanalyse von Straßennetzen (ESN) - Erfahrungen mit den Verfahren der Abschnittsbildung. In: Straßenverkehrstechnik, 52 (9), S. 515-527 


\section{GDV}

DIE DEUTSCHEN VERSICHERER

\section{Gesamtverband der Deutschen Versicherungswirtschaft e.V.}

Wilhelmstraße 43/43G, 10117 Berlin

Postfach 0802 64, 10002 Berlin

Tel.: 030/2020 - 50 00, Fax: 030/20 20-6000

www.gdv.de, www.udv.de 